\title{
Plasmid DNA nanogels as photoresponsive materials for multifunctional bio- applications
}

${\text { Diana } \operatorname{Costa}^{1} \text {, Artur J. M. Valente }}^{2}$ and João Queiroz ${ }^{1}$

${ }^{1}$ CICS-UBI - Health Sciences Research Centre, University of Beira Interior, Av. Infante D. Henrique, 6200-506 Covilhã, Portugal

${ }^{2}$ CQC, Department of Chemistry, University of Coimbra, 3004-535 Coimbra, Portugal

Corresponding author:

Diana Rita Barata Costa

Avenida Infante D. Henrique,

Faculdade de Ciências da Saúde

Universidade da Beira Interior

6200-506 Covilhã

Portugal

E-mail address: dcosta@fcsaude.ubi.pt

Tel.: +351275329002

Fax: +351275329099

\begin{abstract}
This study provides a detailed description on the synthesis and characterization of novel polyamine plasmid DNA nanogels. Ethylene glycol diglycidyl ether was used as crosslinker, in conjugation with polyamines to promote pDNA condensation. The biocompatible nanovectors exhibit a unique swelling behaviour in water and salt solutions. These systems are light photodegradable allowing their use in a broad range of biotechnological applications. Different plasmids, pVAX1-LacZ and pcDNA3-
\end{abstract}


FLAG-p53, and anticancer drugs were, thus, efficiently loaded in the nanogels and their controlled release was demonstrated. Furthermore, the dual delivery of pcDNA3FLAG-p53 gene and anticancer drugs illustrates the possibility of the combination of chemical and gene therapies. This new versatile and easy method of nanohydrogels preparation provides a potential synthetic approach for the design of tunable systems which can display multiple functions, sensitivity to different stimuli and exhibit programmed responses as well.

Keywords: non-viral vectors; plasmid DNA; nanogels; swelling behaviour; co-delivery.

\section{Introduction}

The increasing development in the biotechnological field paid considerable attention to nanogels (NGs), as they can combine properties of colloidal dispersions with features of their macroscopic analogs. The swelling behaviour of NGs is ruled by the osmotic pressure difference inside and outside the gel, and can be triggered by parameters such as charge density or cross-linker density (He et al., 2009 and Tamura et al., 2012). They can dramatically change their degree of swelling in response to an external stimuli, such as, temperature, light or electric field, $\mathrm{pH}$, ionic strength, solvent composition, or added solutes, e.g., ionic surfactants (Borsos and Gilanyi, 2011; Kang et al., 2011 and Ramos et al., 2012). Therefore, NGs have astonishing biomedical applications, such as controlled drug and gene release systems, in vivo biological sensors/imaging diagnostics, scaffolds in regenerative medicine and as suitable reaction sites, among others (Yamane et al., 2009; Wu et al., 2010; Larsson et al., 2014 and Uthaman et al., 2014). Biodegradable NGs, in particular, have the advantage of particle disintegration ensuring the release of the constituent polymer network (Larsson et al., 2014). This performance is of huge interest in drug/gene delivery issues, as NGs can entrap 
bioactive molecules and release them in a controlled and sustained manner enhancing their therapeutic efficacy (Chen et al., 2013 and Lee et al. 2013).

We made use of our knowledge and experience on degradable plasmid DNA macro and microgels (Costa et al., 2011, 2012 and 2014) to design and develop cross-linked polyamine pDNA NGs. The polyamines spermine (SP), protamine sulfate (PS) and polyethylenimine (PEI) were employed to promote pDNA condensation into nanometer particles. This approach has been used as a platform for the formulation of gene delivery vehicles (Bremner et al., 2001; Kircheis et al., 2001; Duan et al., 2012 and Cao et al., 2013). In this work, for the first time for the best of our knowledge, novel polyamine plasmid DNA nanohydrogels have been synthesized by the combination of pVAX1-LacZ or pcDNA3-FLAG-p53 condensation and cross-linking reaction with ethylene glycol diglycidyl ether. Their suitability for the controlled release of mono or dual delivery of biomolecules and their potential for biosensing or bio-patterning technological uses deeply increase their likelihood for clinical translation.

\section{Materials and Methods}

2.1 Materials. Ethylene glycol diglycidyl ether (EGDE) was obtained from Aldrich. $N, N, N^{\prime}, N^{\prime}$-tetramethylethylenediamine (TEMED), sodium chloride $(\mathrm{NaCl})$, spermine tetrahydrochloride (SP), $\mathrm{M}_{\mathrm{W}}: 348.18 \mathrm{~g} \mathrm{~mol}^{-1}, 5.1 \mathrm{kDa}$ protamine sulfate salt (PS) from salmon, doxorubicin (DOX) and epirubicin (EPIR) hydrochlorides and paclitaxel (PTX) were obtained from Sigma. Linear $25 \mathrm{kDa}$ polyethylenimine (PEI) was obtained from Polysciences (Eppenheim, Germany). The pVAX1-LacZ plasmid was from Invitrogen and pcDNA3-FLAG-p53, plasmid 10838, from Addgene (Cambridge, MA, USA). All solutions were freshly prepared using water ultra-pure grade, purified with a Milli-Q system from Millipore (Billerica, MA, USA). Normal Human Dermal Fibroblast 
(NHDF) adult donor cells, Ref. C-12302 (cryopreserved cells) were purchased from PromoCell.

2.2 Plasmid production and purification. The $6.1 \mathrm{kbp} p V A X 1-L a c Z$ and the $6.07 \mathrm{kbp}$ plasmid pcDNA3-FLAG-p53 used in the experiments were amplified in a cell culture of E.coli DH5 $\alpha$, as described elsewhere (Costa et al., 2014). Cells were recovered by centrifugation and the plasmids purified using the Qiagen Plasmid Maxi Kit (Qiagen, CA, USA) according to the supplier's protocol. The protocol is based on a modified alkaline lysis procedure. Following lysis, binding of pDNA to the Qiagen anionexchange resin is promoted under appropriate low-salt and $\mathrm{pH}$ conditions. Impurities are removed by a medium-salt wash and plasmid DNA is eluted in a high salt buffer. The plasmid sample is then concentrated and desalted by isopropanol precipitation and resuspended in Tris- $\mathrm{HCl}$ buffer ( $\mathrm{pH} 8.0$ ). For all plasmids, the 260/280 $\mathrm{nm}$ absorbance ratio was found to be above 1.8 and, also spectrophotometrically, it was confirmed that $\mathrm{A}_{320}$ was negligible suggesting the absence of proteins.

2.3 Preparation of plasmid DNA nanogels. Nanogels have been prepared by two different experimental protocols. In protocol I, aqueous pVAX1-LacZ or pcDNA3FLAG-p53 plasmid solution (2 $\mu \mathrm{g} \mathrm{m} \mathrm{ml}^{-1}$ ), spermine or protamine sulfate or polyethylenimine were mixed together to make a solution A. The ratio between pDNA and spermine or protamine sulfate was equal to one, $\mathrm{R}=[\mathrm{DNA}] /\left[\mathrm{C}^{+}\right]$, where $\left[\mathrm{C}^{+}\right]$is the concentration of the corresponding cationic agent; note that concentrations are determined per charge. Three different ratios of PEI to pDNA have been tested $(4.8,6$ and 10). Solution A was then gently added, drop-wise via a Pasteur pipette to an aqueous solution containing $\mathrm{X} \%$ (where $\mathrm{X}=0.08,0.1$, or 0.15 ) EGDE and TEMED, which is placed in a round-bottom flask in a water bath at $35^{\circ} \mathrm{C}$. The cross-linking 
reaction proceeds for 20 minutes, with a gel sphere being created from each A solution droplet. At the final, $100 \mu 1$ of pDNA nanogels are formed.

In protocol II, pVAX1-LacZ or pcDNA3-FLAG-p53 plasmid solution $\left(2 \mu \mathrm{g} \mathrm{ml}^{-1}\right)$ were chemically cross-linked by $\mathrm{X} \%$ (where $\mathrm{X}=0.08,0.1$ or 0.15 ) EGDE, in the presence of TEMED, for 20 minutes in a water bath at $35^{\circ} \mathrm{C}$. During that time, $25 \mu 1$ of spermine or protamine sulfate were added drop-wise using a Pasteur pipette. Cross-linking and pDNA polyamine induced compaction take place simultaneously to form pDNA nanogels. Following any of the two protocols described, pDNA NGs were washed in $250 \mathrm{mM}$ sodium phosphate buffer at $\mathrm{pH} 7.4$ several times and dried under vacuum.

2.4 Size, polydispersity index and zeta potential measurements. The mean diameter of NGs and the polydispersity index were determined by dynamic light scattering (photon correlation spectroscopy, PCS) using an N5 Particle Analyzer (Beckman Coulter Inc., USA). For these experiments, pDNA gel particles were diluted in $500 \mu 1$ of milliQ ultrapure water as a dispersant medium, followed by 20 minutes incubation at room temperature prior to analysis. Diameters of the NGs were measured at $25^{\circ} \mathrm{C}$ and data collected at $90^{\circ}$ scattering angle. The autocorrelation functions were transformed into intensity-weighted distributions of the apparent hydrodynamic diameter using the available Malvern PCS software version 6.20.

Measurement of NGs average size and the surface charge (zeta potential) were carried out, at $25{ }^{\circ} \mathrm{C}$, in a Zetasizer nano ZS instrument using a zeta dip cell. Dynamic light scattering using a He-Ne laser $633 \mathrm{~nm}$ with non-invasive backscatter optics (NIBS) and electrophoretic light scattering using a patented laser interferometric technique named M3-PALS (Phase analysis Light Scattering) were applied for particle size and zeta potential determination, respectively. The Malvern zetasizer software version 6.34 was 
used. The average values of size and zeta potential were calculated with the data obtained from three independent measurements.

2.5 Determination of encapsulation efficiency. Determination of encapsulation efficiency was performed as described elsewhere (Costa et al., 2014).

2.6 Scanning Electron Microscopy Imaging. Scanning Electron Microscopy (SEM) was used to evaluate the surface morphology of pDNA nanogels. Freshly pDNA carriers were centrifuged $\left(10.000 \mathrm{~g}, 20 \mathrm{~min} ., 25^{\circ} \mathrm{C}\right)$ and the pellet recovered. The pellet was suspended in a solution containing $20 \mu 1$ deionized water with $20 \mu 1$ tungsten salt. $10 \mu \mathrm{l}$ of the recently formed solution was set in roundly shaped cover-slip and left at room temperature overnight to dry. In the following day, the samples were mounted on aluminum supports, fixed with double-sided adhesive tape and sputter coated with gold using an Emitech K550 (London, England) sputter coater. The samples were analyzed using a Hitachi S-2700 (Tokyo, Japan) scanning electron microscope operated at an accelerating voltage of $20 \mathrm{kV}$ at various magnifications.

\subsection{Determination of pDNA nanogels cytotoxicity by 3-[4,5-dimethyl-thiazol-2-yl]-} 2,5-diphenyltetrazolium bromide (MTT) assay. Determination of pDNA nanogels cytotoxicity was performed as described elsewhere (Costa et al., 2012). Plasmid DNA nanogels were applied to a 96-well plate (Nunc.). Before cell seeding, the plates were ultraviolet irradiated for 30 minutes. Human fibroblast cells were plated at a density of $1 \times 10^{4}$ cells per well in 96 well plate at $37^{\circ} \mathrm{C}$ in $5 \% \mathrm{CO}_{2}$ humidified atmosphere, for 24 and 48 hours. After incubation, the redox activity was assessed through the reduction of the MTT. $100 \mu \mathrm{l}$ of MTT dye solution ( $0.05 \mathrm{mg} \mathrm{ml}^{-1}$ in Krebs) were added to each well, 
followed by incubation for 2 hours at $37^{\circ} \mathrm{C}$, in a $5 \% \mathrm{CO}_{2}$ atmosphere. The medium was aspirated and cells were treated with $50 \mu \mathrm{l}$ of isopropanol/ $\mathrm{HCl}(0.04 \mathrm{M})$ for 30 minutes. Absorbance at $570 \mathrm{~nm}$ was measured using a Biorad Microplate Reader Benchmark. The relative cell viability (\%) related to control wells was calculated by $[\mathrm{A}]$ test / $[\mathrm{A}]$ control $\mathrm{x} 100$, where $[\mathrm{A}]$ test is the absorbance of the test sample and $[\mathrm{A}]$ control is the absorbance of the control sample.

2.8 Determination of swelling degree. To study the swelling behaviour of the pDNA NGs, they were freeze dried, collected as a pellet and weighed after being pre-swollen in a $\mathrm{NaOH} 1 \mathrm{mM}$ solution (reference state). The pellet was then placed into distilled water $(10 \mathrm{ml})$ and, in another experiment, into $\mathrm{NaCl}$ solutions at different concentrations $(5,10,15,20,25,30,35,40,45$ and $50 \mathrm{mM})$. The swelling ratio was calculated by dividing the weight of the gels at steady-state swelling by their weight in the reference state.

2.9 Nanogel degradation by ultraviolet light. The pDNA nanogels, immersed in 10 mM Tris- $\mathrm{HCl} \mathrm{pH} 8.0$ buffer, were irradiated with light $(400 \mathrm{~nm})$ using a mercury lamp, in order to promote the nanogel photodisruption and consequent release of pDNA.

2.10 Plasmid DNA release measurements. To determine the amount of pDNA released, a method described elsewhere has been used (Costa et al., 2011). Plasmid DNA released into the supernatant was quantified spectrophotometrically by measuring the absorbance at $260 \mathrm{~nm}$ using a Nanophotometer ${ }^{\mathrm{TM}}$ (Implen, Germany). 
2.11 Encapsulation and release of drugs. DOX, EPIR and PTX have been incorporated into pDNA nanogels by imbibition, as described elsewhere (Costa et al., 2014). The incorporation of the drugs (3 wt.\%) into NGs occurs in dark conditions. DOX, EPIR and PTX concentrations were measured using quartz cuvettes and an ultraviolet-visible spectrophotometry at $480 \mathrm{~nm}$ for the first two drugs and at $227 \mathrm{~nm}$ for PTX. The drug encapsulation (EE) and loading efficiencies (LE) were calculated by using the equations:

$\mathrm{EE}(\%)=[($ total amount of drug - non-bound drug $) /$ total amount of drug $] \times 100$

The loading efficiency (LE) has been determined by using the equation:

$\mathrm{LE}=\left[(\right.$ total amount of drug - non-bound drug $\left.) / \mathrm{m}_{\mathrm{ng}}\right] \times 100$, where $\mathrm{m}_{\mathrm{ng}}$ is the mass of pDNA NG.

\section{Results and Discussion}

3.1 Formation and characterization of polyamine pDNA based nanogels. Plasmid DNA nanogels have been formed by two different synthesis protocols. In protocol I, pVAX1-LacZ or pcDNA3-FLAG-p53 plasmids have been condensed by polyamines (spermine, protamine sulfate or polyethylenimine) and, then, cross-linked with EGDE. In Protocol II, plasmids were first chemically cross-linked with EGDE followed by the addition of spermine or protamine sulfate. In this situation, cross-linking and pDNA condensation occur simultaneously producing pDNA nanogels. Supported by the experience of our group in the formulation of pDNA networks (Costa et al., 2011, 2012 and 2014), here we report a new and original approach: the combination of polyamine condensation and chemical cross-linking, as a route for the synthesis of NGs. Such approach brings some advantages (e.g., the drastic reduction in the size of the produced gel particles) mainly due to polyamines. The ability of natural polyamines to bind DNA 
and to pack it into small dense particles is widely recognized and can be employed in the formulation of transporting agents for the delivery of nucleic acids (Bremner et al., 2001; Kircheis et al., 2001; Duan et al., 2012 and Cao et al., 2013). Additionally, polyamines have amino groups with different $\mathrm{pK} a$ values which prevents endosomal acidification protecting nucleic acids from nucleases digestion, while, facilitating its escape from endosome (Bremner et al., 2001). On the other hand, ethylene glycol diglycidyl ether is a bifunctional cross-linker with epoxide structure; epoxides covalently bind to the guanine bases of the DNA molecule (Eriksson et al., 1993). $N, N, N^{\prime}, N^{\prime}$,-tetramethylethylenediamine (TEMED) was added as an initiator of the cross-linking reaction. The reaction mechanism $\left(S_{N} 2\right)$ of gel formation seems to involve the guanine nitrogen atom at position seven (N-7), which attacks the more substituted carbon of the epoxide or the least hindered end of the epoxide (Benasutti et al., 1988). Following both protocols, spermine (SP) and protamine sulfate (PS) pVAX1-LacZ NGs with $0.08 \%, 0.1 \%$ and $0.15 \%$ cross-linker densities were formed. Testing different cross-linker densities can give valuable information concerning the effect of this parameter on NG properties such as, size, morphology, polydispersity index (PI), swellability and colloidal stability; it also, allows for enhanced design of cross-linked systems. All the prepared nanogels present a spherical or ellipsoidal geometry with diameter sizes ranging from 80 to $400 \mathrm{~nm}$, what make them appropriate for cell uptake and internalization in practical applications concerning gene delivery. From SEM images (Supplementary data, Figure 1S) we can, already, denote differences in the size of NGs between the two synthesis protocols, with protocol I producing gels with smaller sizes for each cross-linker density used. To examine the mean size and polydispersity index, photon correlation spectroscopy experiments, using N5 Particle Analyzer, were carried out for spermine and protamine sulfate NGs prepared by the two 
different protocols. The polydispersity index, among other parameters that will be discussed later, is shown in Table 1. For all systems studied, following the synthesis protocol I leads to both, smaller NG size and PI. The size and polidispersity of gel particles reflected and is, primarily, controlled by the initial size distribution of A solution droplets used in gel preparation (see Experimental section). Moreover, it seems that the strategy to condense pDNA with polyamines, prior to the chemical crosslinking reaction, results in smaller sized nanoscale networks. The cross-linker density plays, also, a role on NG size properties, with highest cross-linked spermine or protamine sulfate NGs presenting smaller sizes and showing higher monodispersity. Furthermore, we found that independently of the synthesis protocol used, protamine sulfate pVAX1-LacZ nanogels always exhibit smaller (ca. $30 \%$ ) sizes when compared with the correspondent cross-linked spermine gels. Polyamines, being positively charged agents, interact predominantly by electrostatic interactions with DNA, inducing their compaction, aggregation and precipitation (Bloomfield et al., 1996 and Korolev et al., 2010). Spermine, a small linear polyamine, has four positive charges, while the protein protamine sulfate presents 21 positive charges, fact that can predict a larger pVAX1-LacZ compaction by the last agent and explains the observed size difference. We should note that the charge ratio between pVAX1-LacZ and spermine or protamine sulfate was equal to 1 and it is possible that a larger ratio is required for the pDNA compaction by spermine. Concerning the interaction between DNA and positivelycharged agents, it has been stated that only when longer polycations were used, the mixing ratio for compaction between positive and negative charges was 1 (Mel'nikova 2000) or below (Minagawa et al., 1991). This may be justified by the flexibility of spermine structure, being thus less efficient in promoting bridging effects between parts of the chains far apart, contrary to what may happen with protamine sulfate chains. All 
of these observations concerning SP and PS pVAX1-LacZ NGs size have been confirmed by an independent experiment using a Zetasizer nano ZS (Table 1). Additionally to particle size, the surface potential of the nanosystem has also been determined. Table 1 presents the zeta potential values for all the EGDE densities spermine and protamine sulfate NGs, prepared by both synthesis protocols. All nanoparticulated gels exhibit positive zeta potential ranging from $+14 \mathrm{mV}$ to $+48 \mathrm{mV}$, approximately. No significant differences, concerning surface charges, were found between the two gel preparation protocols. As expected, higher zeta potential values were found for protamine sulfate NGs. Despite the fact that gels were prepared under charge neutrality conditions, all SP and PS gels are overcharged, what may indicate that SP and PS are unevenly distributed within the pDNA gel and preferentially located at the surface giving rise to this effect. In gene delivery purposes, excess positive charges enable the interaction with anionic proteoglycans at the cell surface promoting vector transportation by endocytosis, with subsequent events until pDNA reaches the nucleus. Table 1 presents also the pDNA loading efficiencies for spermine and protamine sulfate pVAX1-LacZ based nanosystems, prepared with $2 \mu \mathrm{g} \mathrm{ml} \mathrm{m}^{-1}$ initial pDNA loading amount. The pDNA loading efficiencies vary between $46 \%$ and $56 \%$. Smaller efficiencies are observed for the $0.15 \%$ EGDE cross-linked gels. A decrease in this parameter was, also, observed when the protocol II was followed (see section 2.3). The pDNA loading efficiencies achieved here for all pDNA vectors are similar to those reported for similar EGDE cross-linked pDNA hydrogel systems (Costa et al., 2014). We are, thus, aware that improvements must be made on synthesis procedure in order to increase pDNA loading efficiency. To determine the NGs cytotoxicity, the 3-[4,5dimethyl-thiazol-2-yl]-2,5-diphenyltetrazolium bromide (MTT) assay was performed. The MTT assays were performed at 1 and 2 days after NHDF cells having been seeded 
on top of the different pDNA NGs. Our results at day two, Table 1, suggest that, independently of gel formation protocol, all nanogels are non-toxic and therefore suitable for delivery applications in real biological systems. To advance our research on cross-linked nanosystems, polyethylenimine pcDNA3-FLAG-p53 encoding plasmid nanogels were prepared by using experimental protocol I. Cross-linked PEI nanogels were prepared varying the EGDE density $(0.08 \%, 0.1 \%$ and $0.15 \%)$ and at three different N/P ratios (4.8, 6 and 10). Spherical or oval nanoparticle gels were obtained, with diameters ranging from 81 to $109 \mathrm{~nm}$, approximately (Supplementary data, Figure 2S). PEI p53 nanosystems present gel sizes similar to those displayed by protamine sulfate carriers (Supplementary data, Table 1S). Since the major force behind DNA condensation is electrostatic in origin, the number of positive charges of the condensing agent plays a relevant role in mediating this phenomenon. This finding can, also, be related with a stronger interaction with pDNA promoted by the larger charge density of the polycations when compared with spermine. As verified for SP and PS systems, the increase in EGDE density leads to a lowering of the NGs size; varying the N/P ratio of PEI p53 NGs has no significant influence on their size. Consequently, the size of NGs is controlled by two different factors: the greater electrostatic interaction between both protamine sulfate and polyethylenimine with pDNA, and the the cross-linker density. This relevant achievement reflects the versatility of the designed systems and gives us the possibility to manipulate their intrinsic properties in order to improve their performance in specific biomedical applications. As the amount of polyethylenimine in the PEI p53 complexes increases, also increases the zeta potential values, which are in agreement with an increment in the positive nitrogen PEI charges to pDNA negative phosphate ones. For all EGDE densities, considerations concerning the pcDNA3FLAGp53 loading efficiency can be made based on the different N/P ratios of the NGs. 
A lower N/P ratio indicates the presence of a higher pDNA extent and, as expected, the encapsulation of larger pDNA amounts is less efficient and can explain the observed pattern. Furthermore, it was crucial to evaluate the cytotoxic profile of PEI p53 networks. The analysis of dehydrogenase activity in NHDF cells, after two days of incubation with PEI p53 formulations, indicates the cell viability of these systems (Supplementary data, Table 1S).

3.2 Swelling behaviour of polyamine nanogels. We studied the water swelling kinetics of $0.08 \%, 0.1 \%$ and $0.15 \%$ EGDE cross-linked spermine and protamine sulfate pVAX1LacZ nanogels, prepared by synthesis protocol I, as shown in Figure 1A and 1B. In pure water, both SP and PS NGs considerably swell up to an equilibrium point. From a considerable low swelling ratio in the first 30 minutes, the water uptake sharply increases and then begins to level off. For both nanogel systems, the equilibrium swelling was achieved after 3 hours, with spermine NGs exhibiting a larger swelling ability, for all EGDE densities. To elucidation on the diffusion mechanism, swelling kinetics data were fitted to the Weibull function (Costa and Sousa Lobo, 2001).

$$
\frac{Q_{t}-Q_{0}}{Q_{\infty}-Q_{0}}=1-\exp \left[-(t / a)^{b}\right]
$$

where $Q_{\mathrm{t}}$ is the swelling degree of gels at time t, and $Q_{\infty}$ and $Q_{0}$ are the swelling degree at equilibrium and reference states, respectively. The computed fitting parameters $a$ and $b$ are shown in supplementary data, Table $2 \mathrm{~S}$. The shape parameter $b$ is, for all systems, higher than 1, characterizing a S-shaped sorption, which normally occurs in highly swelling systems (Hedenqvist and Gedde, 1999). Moreover the magnitude of $b$ values also indicates that the water sorption follows a Super-Case II mechanism, which is related with processes of relaxation of boundary concentration on the polymer surface; i.e. in the initial stage, the swelling is mostly on one-dimensional because the unswollen 
core suppresses swelling perpendicular to the main flux direction; at later stages, when the core is plasticized by the water, the polymer may swell in all three dimensions (Mazich et al., 1992). The scale parameter, $a$, defines the time scale of the swelling process. For both nanovectors, we assisted to the pronounced role of cross-linker density on the degree of swelling. The swelling ratio for $0.08 \%$ EGDE spermine or protamine sulfate NGs is considerably larger than that of corresponding $0.15 \%$ EGDE gels. An increase in the level of cross-linker agent leads to a larger resistance to the osmotic swelling force and, thus, to a reduced equilibrium swelling and to a slower process (i.e. a higher $a$ value). When SP and PS NGs were placed into salt solutions $(\mathrm{NaCl})$, at different concentrations, Figure 1C and 1D, they shrink due to the screening effect of the salt. In the presence of the salt, the difference between ion concentrations inside and outside the gel is reduced. Consequently, the driving force of swelling decreases gradually with increasing salt concentration. We do confirm the swelling responsiveness of the developed NGs. Moreover, the NG swelling behaviour can be modulated by the addition of an electrolyte, as well as by the cross-linker density used in the NG formation.

3.4 Release of p53 gene and anticancer drugs. The degradation on ultraviolet light exposure (photo-oxidation) of EGDE leads to the removal of the chemical cross-links and can allow the release of the constituent network polymer inducing changes in gel weight, mechanical properties, mesh size, porosity and in the degree of swelling (Costa et al., 2011 and 2014). In order to demonstrate the NG photodisruption, spermine and protamine sulfate pVAX1-LacZ and polyethylenimine pcDNA3-FLAG-p53 gel spheres were irradiated with ultraviolet light at $400 \mathrm{~nm}$ and the changes, induced by light, on average diameter were monitored by dynamic light scattering (Zetasizer nano ZS) over disruption time (Supplementary data, Figure 3S). Another strong evidence of NGs 
photodegradation comes from the release of pVAX1-LacZ from SP and PS networks when gels are irradiated with ultraviolet light (Supplementary data, Figure 4S). To demonstrate the feasibility of drug and gene co-delivery with the designed photoresponsive NGs, both the pcDNA3-FLAG-p53 based plasmid and different anticancer drugs (doxorubicin, epirubicin and paclitaxel) were simultaneously loaded into PEI-nanogels at various N/P ratios. Great drug loading capacities, between $42 \%$ and $62 \%$, were found for all studied vectors. (Supplementary data, Table 3S). Epirubicin is the anticancer drug that can be loaded in larger extent (Supplementary data, Table 3S), no matter the cross-linker density or the N/P ratio considered. Moreover, for all EGDE densities, PEI-p53 NGs with N/P ratio of 4.8 evidenced the largest DOX, EPIR and PTX loading efficiencies. For this fact can contribute the smaller p53 EE at the N/P ratio of 4.8. The smaller pcDNA3-FLAG-p53 content into the network facilitates the uptake of higher drug amounts. The photodegradable property of NGs has been used to promote the controlled release of pcDNA3-FLAGp53 gene and anticancer drugs. Figure 2A shows the release profile of the p53 gene from PEI p53 gels, N/P ratio of 4.8, after their irradiation with UV light. Considerable amounts of pcDNA3-FLAG-p53 can be released over time in an extent that depends on cross-linker density. In the dark, however, insignificant pcDNA3-FLAG-p53 gene can be released from PEI networks. Similar results were obtained for the N/P ratios of 6 and 10 (data not shown). Although in vitro transfection efficiency tests are not reported, preliminary studies with cells of different histological type, demonstrate PEI gels cell uptake and transfection feasibility. Additionally, our previous work on chemical EGDE pDNA gels strongly indicates the integrity and bioactivity of released pcDNA3-FLAGp53 gene encoding plasmid, with pDNA performing its function upon delivery to the cell which results in the p53 protein expression (Costa et al., 2014). Doxorubicin, 
epirubicin and paclitaxel can, also, be efficiently released from PEI NGs under gel matrix photodisruption, for all N/P ratios studied. Figures $2 \mathrm{~B}, 2 \mathrm{C}$ and $2 \mathrm{D}$ present the release curves for DOX, EPIR and PTX, respectively. Less cross-linked PEI nanogels allow for the higher content of released drug; approximately, $89.7 \%$ of DOX, $95.6 \%$ of EPIR and $95.4 \%$ of PTX are released from PEI p53 matrices at N/P ratios of 4.8, 6 and 10, respectively. No significant amounts of drugs were released at dark conditions. Identical profile and percentage drug released were obtained for the other N/P ratios, in each system. Despite our similar results for all N/P ratios on drug and gene release curves, it is possible that cell transfection efficiency varies with N/P ratio. Finding the optimal N/P ratio is crucial to achieve enhanced transfection efficiency. Our current in vitro studies seems to support this assumption. We, thus, demonstrate the co-delivery of pcDNA3-FLAG-p53 gene and different anticancer drugs in a controlled and sustained manner from PEI p53 photoresponsive NGs. This may be particularly useful in cancer therapy due to the possibility of synergetic effect, achieved through the combined action of chemical and gene therapy. In vitro studies concerning this issue are, already, being performed by our research team and will be reported soon. Although there are other groups developing DNA hydrogels for specific applications such as controlled drug delivery, tissue engineering, 3D cell culture, cell transplant therapy and other biomedical uses (Peng et al., 2012 and Song et al., 2013), our team brings novelty with the light triggered phenomenon and the possibility of delivering genes and drugs either alone or as a dual-delivery.

\section{Conclusions}

We developed a novel method to produce polyamine based pDNA nanogels, by the conjugation of pDNA (pVAX1-LacZ or pcDNA3-FLAG-p53) condensation and crosslinking reaction. Biocompatible pDNA gels have suitable sizes, morphology and surface 
charges, as well as, stimuli-responsive swelling behaviour. Their photodegradation property provides a powerful tool for the design of efficient tunable vectors for advanced biomedical applications ranging from potential biosensing/diagnostic issues to controlled mono or dual delivery systems.

\section{Acknowledgments}

We are grateful for financial support from Fundação para a Ciência e a Tecnologia (FCT), (SFRH/BPD/47229/2008) and to PTDC/EBB-Bio/114320/2009. The authors would like to thank to Fani Sousa e Ângela Sousa for the $6.07 \mathrm{kbp}$ plasmid pcDNA3FLAG-p53 production and recovery, and to Prof. ${ }^{a}$ Maria da Graça Miguel (Department of Chemistry, University of Coimbra, Portugal) for fruitful discussions.

\section{References}

Benasutti, M., Ejadi, S., Whitlow, M. D., Loechler, E. L., 1988. Mapping the binding site of aflatoxin B1 in DNA: systematic analysis of the reactivity of aflatoxin B1 with guanines in different DNA sequences. Biochemistry 27, 472-481.

Bloomfield, V. A., 1996. DNA Condensation. Curr. Opin. Struct. Biol. 6, 334-341.

Borsos, A., Gilanyi, T., 2011. Interaction of cetyl-trimethylammonium bromide with swollen and collapsed poly(N-isopropylacrylamide) nanogel particles. Langmuir 27, $3461-3467$.

Bremner, K. H., Seymour, L. W., Pouton, C. W., 2001. Harnessing nuclear localization pathways for transgene delivery. Curr. Opin. Mol. Ther. 3, 170-77.

Cao, M., Deng, X., Su, S., Zhang, F., Xiao, X., Hu, Q., Fu, Y., Yang, B. B., Wu, Y., Sheng, W., Zeng, Y., 2013. Protamine sulfate-nanodiamond hybrid nanoparticles as a vector for MiR-203 restoration in esophageal carcinoma cells. Nanoscale 5, 1212012125. 
Chen, W., Zheng, M., Meng, F., Cheng, R., Deng, C., Feijen, J., Zhong, Z., 2013. In situ forming reduction-sensitive degradable nanogels for facile loading and triggered intracellular release of proteins. Biomacromolecules 14, 1214-1222.

Costa, D., Valente, A. J., Miguel, M. G., Queiroz J., 2011. Gel network photodisruption: a new strategy for the co-delivery of plasmid DNA and drugs. Langmuir 27, 13780-13789.

Costa, D., Queiroz, J., Miguel, M. G., Lindman, B., 2012. Swelling behavior of a new biocompatible plasmid DNA hydrogel. Colloids Surf. B 92, 106-112.

Costa, D., Valente, A. J., Miguel, M. G., Queiroz, J., 2014. Plasmid DNA hydrogels for biomedical applications. Adv. Colloid Interface Sci. 5, 257-264.

Costa, P., Sousa Lobo, J. M., 2001. Modelling and comparison of dissolution profiles. Eur. J. Pharm. Sci. 13, 123-133.

Duan, S-Y., Ge, X-M., Lu, N., Wu, F., Yuan, W., Jin, T., 2012. Synthetic polyspermine imidazole-4, 5-amide as an efficient and cytotoxicity-free gene delivery system. Int. J. Nanomed. 7, 3813-3822.

Eriksson, M., Kim, S. K., Sen, S., Gräslund, A., Jernström, B., Nordén, B., 1993. Location of Excimer-Forming Adducts of (+)-anti-Benzo[a]pyrene Diol Epoxide in DNA. J. Am. Chem. Soc. 115, 1639-1644.

He, J., Tong, X., Zhao, Y., 2009. Photoresponsive nanogels based on photocontrollable cross-links. Macromolecules 42, 4845-4852.

Hedenqvist, M. S., Gedde, U. W., 1999. Parameters affecting the determination of transport kinetics data in highly swelling polymers above $\mathrm{T}_{\mathrm{g}}$. Polymer 40, 2381-2393.

Kang, H., Trondoli, A. C., Zhu, G., Chen, Y., Chang, Y. J., Liu, H., Huang, Y. F., Zhang, X., Tan, W., 2011. Near-infrared light-responsive core-shell nanogels for targeted drug delivery. ACS Nano 5, 5094-5099. 
Kircheis, R., Wightman, L., Schreiber, A., Robitza, B., Rössler, V., Kursa, M., Wagner, E., 2001. Polyethylenimine/DNA complexes shielded by transferrin target gene expression to tumors after systemic application. Gene Ther. 8, 28-40.

Korolev, N., Lyubartsev, A., Nordenskiold, L., 2010. Cation-induced polyelectrolytepolyelectrolyte attraction in solutions of DNA and nucleosome core particles. Adv. Colloid Interface Sci. 158, 32-47.

Larsson, M., Bergstrand, A., Mesiah, L., Van Vooren, C., Larsson, A., 2014. Nanocomposites of Polyacrylic Acid Nanogels and Biodegradable Polyhydroxybutyrate for Bone Regeneration and Drug Delivery. J. Nanomater. DOI: doi.org/10.1155/2014/371307.

Lee, J., Jeong, D., Seo, S., Na, K., 2013. Biodegradable nanogel based on all-trans retinoic acid/pullulan conjugate for anti-cancer drug delivery. J. Pharm. Inv. 43, 63-69. Lim, C. K., Singh, A., Heo, J., Kim, D., Lee, K. E., Jeon, H., Koh, J., Kwon, I. C., Kim, S., 2013. Gadolinium-coordinated elastic nanogels for in vivo tumor targeting and imaging. Biomaterials 28, 6846-52.

Mazich, K. A., Rossi, G., Smith, S. A., 1992. Kinetics of solvent diffusion and swelling in a Model Elastomeric System. Macromolecules 25, 6929-6933.

Mel'nikova, Y. S., 2000. Association of amphiphiles and polyelectrolytes with DNA. Doctoral thesis, Lund University, Lund, Sweden.

Minagawa, K., Matsuzawa, Y., Yoshikawa, K., Matsumoto, M., Doi, M., 1991. Direct observation of the biphasic conformational change of DNA induced by cationic polymers. FEBS Lett. 295, 67-69.

Peng, S., Lee, J. B., Yang, D., Roh, Y. H., Funabashi, H., Park, N., Rice, E. J, Chen, L., Long, R., Wu, M., Luo, D., 2012. A mechanical metamaterial made from a DNA hydrogel. Nat. Nanotechnol. 109, 816-820. 
Ramos, J., Imaz, A., Forcada, J., 2012. Temperature-sensitive nanogels: poly(Nvinylcaprolactam) versus poly(N-isopropylacrylamide). Polym. Cem. 3, 852-856.

Song, L., Ho, V. H. B., Chen, C., Yang, Z., Liu, D., Chen, R., Zhou, D., 2013. Efficient, pH-Triggered Drug Delivery Using a pH-Responsive DNA-Conjugated Gold Nanoparticle. Adv. Healthcare Mater. 2, 275-280.

Tamura, G., Shinohara, Y., Tamura, A., Sanada, Y., Oishi, M., Akiba, I., Nagasaki, Y., Sakurai, K., Amemiya, Y., 2012. Dependence of the swelling behavior of a pHresponsive PEG-modified nanogel on the cross-link density. Polym. J. 44, 240-244.

Uthaman, S., Maya, S., Jayakumar, R., Cho, C. S., Park, I. K., 2014. Carbohydratebased nanogels as drug and gene delivery systems. J. Nanosci. Nanotechnol. 14, 694704.

Wu, W., Zhou, T., Berliner, A., Banerjee, P., Zhou, S. Q., 2010. Smart core-shell hybrid nanogels with Ag nanoparticle core for cancer cell imaging and gel shell for $\mathrm{pH}$ regulated drug delivery. Chem. Mater. 22, 1966-1976.

Yamane, S., Sugawara, A., Watanabe, A., Akiyoshi, K., 2009. Hybrid Nanoapatite by Polysaccharide Nanogel-templated mineralization. J. Bioact. Compat. Polym. 24, 151168.

\section{Figure Captions}

Figure 1. Water swelling kinetics (A, B) and swelling behaviour in salt solutions (C, D), at different concentrations, of $0.08 \%(\bullet), 0.1 \%(\Delta)$ and $0.15 \%(\boldsymbol{\bullet})$ EGDE crosslinked spermine and protamine sulfate pVAX1-LacZ nanogels, prepared by synthesis protocol I.

Figure 2. Cumulative release of pcDNA3-FLAG-p53 gene, doxorubicin (DOX), epirubicin (EPIR) and paclitaxel (PTX) from $0.08 \%(\bullet), 0.1 \%(\Delta)$ and $0.15 \%(\bullet)$ EGDE cross-linked PEI p53 nanogels at N/P ratio of 4.8 (A) and (B), 6 (C) and 10 (D), 
respectively, after their irradiation with UV light $(400 \mathrm{~nm})$ and in the dark conditions as a function of time.

\section{Figures}
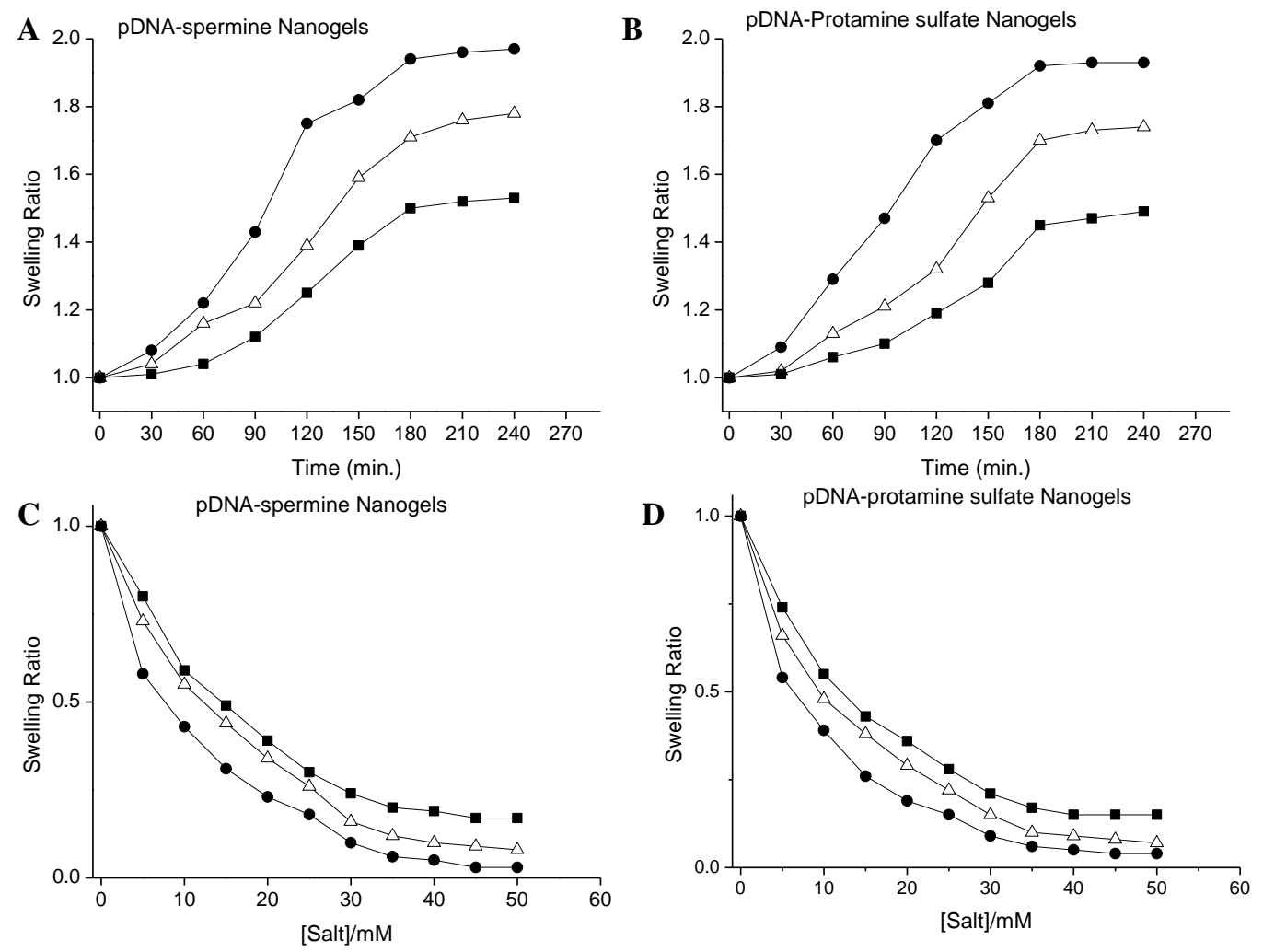

Figure 1. 

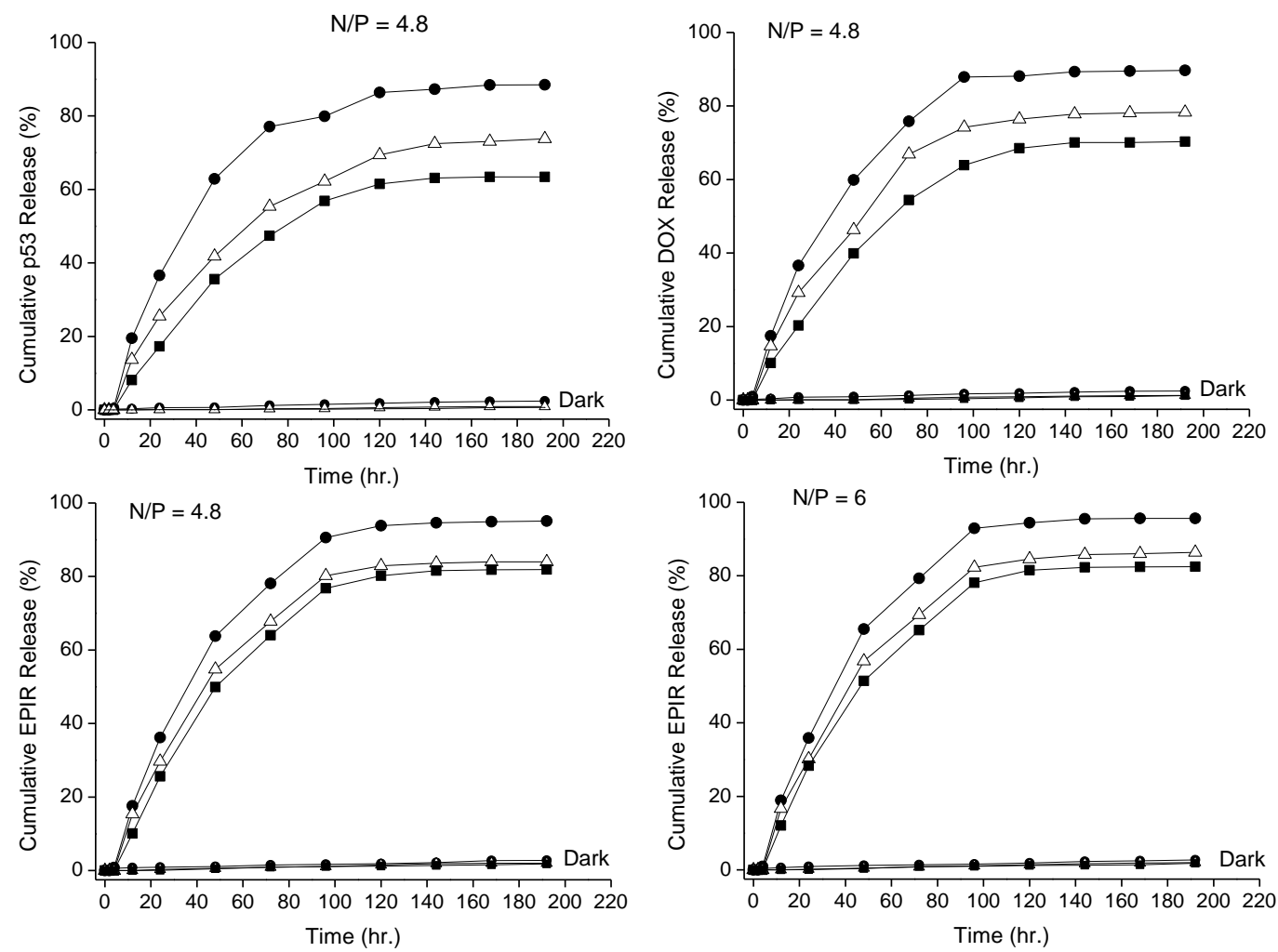

Figure 2.

\section{Tables}

Table 1. Summary of the properties of spermine (SP) and protamine sulfate (PS) pVAX1-LacZ cross-linked nanogels synthesized by Protocol I (A) and Protocol II (B). In all cases, pVAX1LacZ initial loading amount was $2 \mu \mathrm{g} \mathrm{ml}^{-1}$. The values of the different parameters were calculated with the data obtained from three independent measurements (mean $\pm S D, n=3$ ). Mean size determined by using the Zetasizer nano ZS.

\begin{tabular}{lccccc}
\hline A $\quad$ System & pDNA LE $(\%)$ & Mean size/nm & Zeta Potential (mV) & P.I. & Cell Viability (\%) \\
\hline pDNA-SP-0.08\% EGDE & $54 \pm 1$ & $178 \pm 6$ & $19 \pm 0.1$ & $0.39 \pm 0.02$ & 88 \\
pDNA-SP-0.1\% EGDE & $53 \pm 1.1$ & $140 \pm 3$ & $18 \pm 1.0$ & $0.34 \pm 0.31$ & 86 \\
pDNA-SP-0.15\% EGDE & $50 \pm 1$ & $121 \pm 1$ & $14 \pm 0.6$ & $0.31 \pm 0.01$ & 81 \\
\hline pDNA-PS-0.08\% EGDE & $56 \pm 1$ & $127 \pm 3$ & $41 \pm 0.2$ & $0.24 \pm 0.30$ & 89 \\
pDNA-PS-0.1\% EGDE & $55 \pm 1$ & $100 \pm 1$ & $40 \pm 0.5$ & $0.20 \pm 0.08$ & 87 \\
pDNA-PS-0.15\% EGDE & $52 \pm 0.9$ & $89 \pm 1$ & $45 \pm 0.1$ & $0.12 \pm 0.05$ & 83 \\
\hline
\end{tabular}




\begin{tabular}{lccccc}
\hline B System & pDNA LE $(\%)$ & Mean size $/ \mathrm{nm}$ & Zeta Potential $(\mathrm{mV})$ & P.I. & Cell Viability (\%) \\
\hline pDNA-SP-0.08\% EGDE & $46 \pm 1$ & $389 \pm 15$ & $18 \pm 0.3$ & $0.91 \pm 0.40$ & 87 \\
pDNA-SP-0.1\% EGDE & $48 \pm 1$ & $359 \pm 20$ & $16 \pm 0.7$ & $0.89 \pm 0.03$ & 85 \\
pDNA-SP-0.15\% EGDE & $48 \pm 0.2$ & $346 \pm 12$ & $14 \pm 0.4$ & $0.88 \pm 0.01$ & 81 \\
\hline pDNA-PS-0.08\% EGDE & $50 \pm 0.7$ & $280 \pm 15$ & $48 \pm 0.6$ & $0.56 \pm 0.02$ & 88 \\
pDNA-PS-0.1\% EGDE & $52 \pm 0.3$ & $202 \pm 11$ & $45 \pm 0.5$ & $0.54 \pm 0.21$ & 85 \\
pDNA-PS-0.15\% EGDE & $53 \pm 0.1$ & $189 \pm 9$ & $46 \pm 0.2$ & $0.48 \pm 0.09$ & 80 \\
\hline
\end{tabular}

